\title{
Early Sleep Apnea Detection and Treatment in Stroke Rehabilitation
}

\author{
Lisandro E. Olmos ${ }^{\mathrm{a}, \mathrm{c}}$, Melania Ron ${ }^{\mathrm{a}}$, Valeria Grondona ${ }^{\mathrm{a}}$, \\ Matias Gianella ${ }^{\mathrm{a}}$, Sebastian F. Ameriso ${ }^{\mathrm{b}}$
}

\begin{abstract}
Background: To assess whether the presence of obstructive sleep apnea is associated with changes in the motor, cognitive and functional outcomes in stroke patients. To investigate whether early intervention of obstructive sleep apnea impact the outcomes.
\end{abstract}

Methods: We performed overnight polysomnography in 25 patients admitted to the stroke rehabilitation unit. Those with an apnea/hypopnea index (AHI) > 10 per hour were randomized to standardized 30 day rehabilitation program with or without the addition of $\mathrm{Bi}$ level positive air pressure treatment at night. Cognitive, motor and functional status were assessed at baseline and at 30 days.

Results: The early Bi Level Possitive Air Pressure treatment in the obstructive sleep apnea group $(n=13)$ produced improvements of the motor scores compared to patients who did not receive it $(\mathrm{n}=$ 12). The group with BiPAP treatment showed significant improvements in the The Fugl-Meyer Upper Extremity Motor Assessment score $(p=0.05)$ and the The Fugl-Meyer Lower Extremity Motor Assessment score $(p=0.019)$ compared with the group without $\mathrm{Bi}$ PAP treatment.

Conclusions: Early obstructive sleep apnea detection and treatment suggest that BiPAP treatment plays a role in motor recovery of stroke patients. The observations suggest that polysomnography evaluation looking for obstructive sleep apnea should be part of the standardized evaluation of stroke patients at admission to stroke rehabilitation facilities.

Manuscript accepted for publication September 30, 2011

${ }^{a}$ FLENI Rehabilitation Institute, Escobar, Buenos Aires, Argentina ${ }^{\mathrm{b}}$ FLENI, Fundacion para la lucha contra las Enfermedades Neurologicas de la Infancia, Instituto de Investigaciones Dr. Raul Carrea, Argentina ${ }^{\mathrm{c} C o r r e s p o n d i n g ~ a u t h o r: ~ R u t a ~} 9$ Km 52,5 Escobar B1625 XAF, Buenos Aires, Argentina. Email: lolmos@fleni.org.ar

doi:10.4021/jnr55w
Keywords: Stroke; Sleep apneas; Rehabilitation

\section{Introduction}

Obstructive sleep apnea (OSA) occurs when mechanisms that maintain pharyngeal patency during sleep are dysfunctional, resulting in narrowing or collapse of the upper airway. Most studies support the hypothesis of an association between OSA and vascular disease. Yaggi el al. [1] demonstrated that OSA significantly increases the risk of stroke or death independently of other risk factors, including hypertension. OSA is linked to metabolic, vascular, hematology, and genetic markers associated with increased cardiovascular disease risk [2]. Treatment options for OSA include postural changes, weight loss, oral devices and mechanical ventilation, with either continuous positive airway pressure (CPAP) or bi level positive airway pressure (BiPAP).

The implications of disturbed sleep, in particular in rehabilitation settings, have recently gained prominence. Sleep breathing disorders are not always apparent and currently, polysomnography (PSG) is not routinely performed in stroke patients during their rehabilitation program. The effects of the early detection and treatment of OSA in this setting remain to be elucidated [3].

OSA is common among patients with stroke [4-6]. It is reported in as many as $44-74 \%$ of patients with stroke [7, 8]. However no guidelines have been developed for the diagnosis and management of this condition in the setting of acute stroke evaluation and treatment [3, 9]. The European Stroke Organization (ESO) guidelines [3] and the American Heart Association do not include PSG as an indication in stroke patients. The PSG Task Force from the American Sleep Disorders Association [10] and the Practice parameters for the indications for PSG procedures [9] do not provide standardized recommendations for detection and management of OSA during acute hospitalization and during the inpatient rehabilitation period of stroke patients.

CPAP and BiPAP treatment improve the quality of life and neurocognitive function, reduce hypersomnia, and lower 
blood pressure in the general sleep apnea population [11]. The presence of OSA in stroke patients has been associated with lower functional capacity at admission and discharge from rehabilitation facilities, and with a longer period of hospitalization [12]. No prospective study has shown that OSA treatment improves cognitive function and activities of daily living (ADL) in stroke patients [11-13]. Previous studies of CPAP treatment in patients with OSA diagnosed after acute stroke provided insight into the compliance of CPAP, the effectiveness of treatment [13, 14], the improvement of depressive symptoms [13], and the decrease of nocturnal blood pressure [14], but failed to show benefits in Barthel Index (BI) and Mini Mental State Examination (MMSE)] [13].

Whether treatment of OSA in this setting improves functional capacity is unknown. Accordingly, the above information provides a strong rationale to conduct a well-designed randomized clinical trial to determine whether treatment of OSA could improve short-term functional outcomes.

The objective of the study is to investigate the effects of early detection and treatment of OSA on short-term motor, cognitive and functional outcomes in stroke patients.

\section{Material and Methods}

The present study was approved by the institutional review board and ethics committee. Every subject was given informed consent. The procedures followed were in accordance with institutional guidelines. This is a single blind prospective randomized trial. Between June 2006 and May 2008, 188 ischemic stroke patients were admitted to FLENI Stroke Rehabilitation Unit. Eligible patients were those admitted from the acute care facilities to the stroke rehabilitation unit within 2 to 8 weeks of stroke onset (mean $4 \pm 2$ weeks), with the following inclusion criteria: (1) 18 to 84 years of age, (2) ischemic stroke confirmed by history of sudden onset of a neurological deficit lasting $>24$ hs, neurological deficit on physical examination, and brain lesion compatible on computerized tomography or MRI, (3) ability to follow commands, (4) diagnosis of OSA on an overnight PSG.

The exclusion criteria included (1) patients who refused to participate in the study; (2) patients without diagnosis of OSA; (3) patients without compliance to BiPAP-CPAP treatment; (4) Subjects admitted $<2$ or $>8$ weeks after the stroke; (5) previous diagnosis of OSA under CIPAP-BiPAP treatment; (6) recurrent stroke; (7) associated traumatic brain injury; (8) dementia; (9) drug or alcohol abuse; (10) psychiatric condition except depression; (11) heart failure; (12) respiratory distress or any other clinical condition that preclude their participation in the trial; (13) aphasia.

All patients received standardized 30 days stroke rehabilitation program that included 4 to 6 hours a day of physical, occupational, cognitive and speech therapies. During the first week of admission, every patients included in the protocol was evaluated by PSG. The apnea-hypopnea Index (AHI) was defined as the frequency of obstructive apneas and hypopneas per hour of sleep. Subjects with an AHI > 10 per hour were randomized to either standardized 30 day rehabilitation program (Group OSA without BiPAP, $\mathrm{n}=12$ ) or rehabilitation program plus BiPAP treatment (Group OSA with BiPAP, $n=13$ ). The BiPAP was autotitrated during the PSG to reduce the (AHI) to $<5$ or to the highest pressure tolerated. A manual titration was also conducted during the first night according with snoring, micro arousal episodes and sleep architecture. The optimal level of positive air pressure for each patient was applied during the entire protocol. Staff members applied the Bipap to the subjects nightly. Patients used the BiPAP respiration for at least 4 hours at night under the nurse surveillance.

The overnight recordings were made in hospital and sampled using a 32-channel Bioscience Polysomnograph equipped with Stellate Harmonie 6.0 software (Canada). Standard techniques for scoring sleep stages and arousals were used [10]. The recordings included nasal and oral airflow, microphone placed on the throat, respiratory movements, oxygen saturation and heart rate by finger (oximetry Nonim USA), respiratory and body movements and position, thoracic and abdominal belts (AcSleep 119 Biolink, Buenos Aires. Argentina), three electromyography channels (mentonian and both tibialis muscles), two electro oculography channels, twelve electrocardiography channels, electroencephalography recording. The CPAP (Autoset Advantage Resmed Spirit corp, Sidney, Australia) titration was performed automatically according with the positive air pressure necessary to decrease the AHI. All recordings were scored manually. The duration of sleep was estimated from the pressure-sensitive bed recordings and from visual observation of patients during the night. The polysomnograms were analyzed by certificated clinicians.

We used the Bipap instead of the CPAP because in preliminary experience patients who need high positive respiratory pressures showed better compliance with Bipap treatment. The BiPAP equipment and the facial mask used were from Resmed Spirit corp.

\section{Assessment scales}

All subjects underwent a baseline and 30 days standard examination including a motor, cognitive and functional neurological condition. The neuropsychological battery consisted in the following tests:

Addenbroke’s Cognitive Examination (ACE) [15]

It is a brief screening test of general cognitive functions which evaluates cognitive domains such as orientation, attention, memory, language, verbal fluency and visuospatial skills. It incorporates the Mini - Mental State Exam.

Frontal Assessment Battery (FAB) [16] 
Table 1. Comparison at Baseline of Treatment and Control Group

\begin{tabular}{lll}
\hline & Obstructive Sleep Apneas & \\
& With BiPAP & Without BiPAP \\
\hline Patients (n) & 13 & 12 \\
Age yrs (Mean) & 60 & 65,5 \\
Range & $55-68$ & $58-72$ \\
Sex & & \\
Male & 8 & 6 \\
Female & 5 & 6 \\
Hypertension & $10(76.9 \%)$ & $10(83.3 \%)$ \\
Hypercholesterolemia & $5(38.4 \%)$ & $2(16.6 \%)$ \\
Smoking & $4(30.7 \%)$ & $4(33.3 \%)$ \\
Diabetes Mellitus & $1(7.7 \%)$ & $4(33.3 \%)$ \\
Coronary heart disease & $5(38.4 \%)$ & $1(8.33 \%)$ \\
Cardiac arrhythmias & $3(23 \%)$ & $1(8.33 \%)$ \\
Ischemic stroke location & $18-41$ & $5-16$ \\
Supratentorial, n (\%) & $12(92.3 \%)$ & \\
Infratentorial, n (\%) & $1(7.7 \%)$ & $10-37$ \\
NIH score & $5-14 \%)$ \\
Mean & & \\
Range & & \\
Body Mass Index & & \\
Mean & & \\
\hline
\end{tabular}

This frontal lobes battery consists of conceptualization and abstract reasoning, mental flexibility, motor programming and executive control of action, resistance to interference, self-regulation, inhibitory control and environmental autonomy.

Digit Span Subtest of the Wechsler Memory Test (forward \& backward) [17]

This test is used to evaluate attention and working memory for verbal material.

The functional neurological condition consisted of the following tests:

The Functional Independence Measure (FIM) consists of 18 items organized under six categories, including: self care; sphincter control; mobility; locomotion; communication; and social cognition [18].
The Barthel Index (BI) included 10 items, including: feeding, transfers, personal grooming and hygiene, bathing, toileting, walking, stair climbing, and controlling bladder and bowel [19].

The motor performance was evaluated by The FuglMeyer motor assessment that consists of 155 items dealing with the shoulder, elbow, forearm, wrist, and hand in the upper extremity and the hip, knee, and ankle in the lower extremity; reflex activity; balance; sensation; position sense and range of motion [20].

\section{Statistical analysis}

The median and the 25-75 percentiles were obtained for both conditions (OSA with BiPAP treatment and OSA 
Table 2. Comparison at Baseline and 30 Days

\begin{tabular}{|c|c|c|c|c|}
\hline & Baseline & Day 30 & $\begin{array}{l}\text { Absolute change } \\
\text { (Day 30-Baseline) }\end{array}$ & p-Value \\
\hline \multicolumn{5}{|l|}{ FIM } \\
\hline OSA w/ Bipap & $43(24.5-50.5)$ & 61 (57-91.5) & 18 & $0.001 *$ \\
\hline OSA & $44(23-49)$ & 70 (38.5-75.7) & 26 & $0.002 *$ \\
\hline \multicolumn{5}{|l|}{ BI } \\
\hline OSA w/ Bipap & $5(0-25)$ & 50 (12.5-57.5) & 45 & $0.001 *$ \\
\hline OSA & $7.5(0-15)$ & 27,5 (5-43.7) & 20 & $0.003 *$ \\
\hline \multicolumn{5}{|l|}{ FMUE } \\
\hline OSA w/ Bipap & $5(4-31)$ & $24(4-47)$ & 19 & $0.018^{*}$ \\
\hline OSA & $4(4-4)$ & $4(4-6.75)$ & 0 & $0,041^{*}$ \\
\hline \multicolumn{5}{|l|}{ FMLE } \\
\hline OSA w/ Bipap & $13(4-21)$ & $21(7-27)$ & 8 & $0.007 *$ \\
\hline OSA & $4(4-5.75)$ & $6(4-12.75)$ & 2 & $0.027^{*}$ \\
\hline \multicolumn{5}{|l|}{ ACE } \\
\hline OSA w/ Bipap & 75 (67-77) & 85 (77-92) & 10 & $0.028 *$ \\
\hline OSA & 80 (71.5-84.7) & 80 (79.2-91.5) & 0 & 0.061 \\
\hline \multicolumn{5}{|l|}{ FAB } \\
\hline OSA w/ Bipap & $10(5.2-14.2)$ & $12.5(8.7-14.5)$ & 2.5 & 0.223 \\
\hline OSA & $11(10-13.75)$ & $14.5(8.7-17)$ & 3.5 & 0.141 \\
\hline \multicolumn{5}{|l|}{ DSF } \\
\hline OSA w/ Bipap & $6(5-7)$ & $6(5-7)$ & 0 & 0.317 \\
\hline OSA & $5(3.5-6.7)$ & $5.5(5-6.7)$ & 0.5 & 0.414 \\
\hline \multicolumn{5}{|l|}{ DSB } \\
\hline OSA w/ Bipap & $4(3-4)$ & $4(3-5)$ & 0 & 0.518 \\
\hline OSA & $3(2,25-4)$ & $3(3-4)$ & 0 & 0.564 \\
\hline
\end{tabular}

Comparison at Baseline and 30 Days in the OSA With BiPAP Group and in the OSA Without BiPAP Treatment (Intragroup Comparison) in the Following Scales: Functional Independence Measure, Barthel Index, Fugl-Meyer Motor Assessment Upper and Lower Extremities, Addenbroke's Cognitive, Examination, Frontal Assessment Battery, Digit Span Subtest of the Wechsler Memory Test. Data are presented as median (percentiles 25-75) unless otherwise stated; * Statistical Significance

without BiPAP). The analysis for the intragroup statistical significance consisted in the comparison 30 days minus baseline in the OSA with BiPAP group and in the OSA without BiPAP treatment. The analysis for the intergroup statistical significance consisted in the comparison between OSA with BiPAP versus OSA without BiPAP treatment. A non-parametric variance analysis of the Kruskall-Wallis test was utilized. The Mann-Whitney test was utilized for the intergroup analysis and the Wilcoxon signed rank test was utilized for the intragroup comparative analysis.

All participants were tested two times for each test (admission and 30 days). Test assignment was randomized to eliminate fatigue bias from test to test. All tests were performed by the same team blind to the PSG results. The physical therapists, occupational therapists and psychologist who performed the evaluations were also not informed about the study hypothesis to avoid any individual bias. Individual results were recorded for further analysis. A p value $<0.05$ was considered statistically significant.

\section{Results}

Twenty-five subjects met criteria and agreed to participate: 


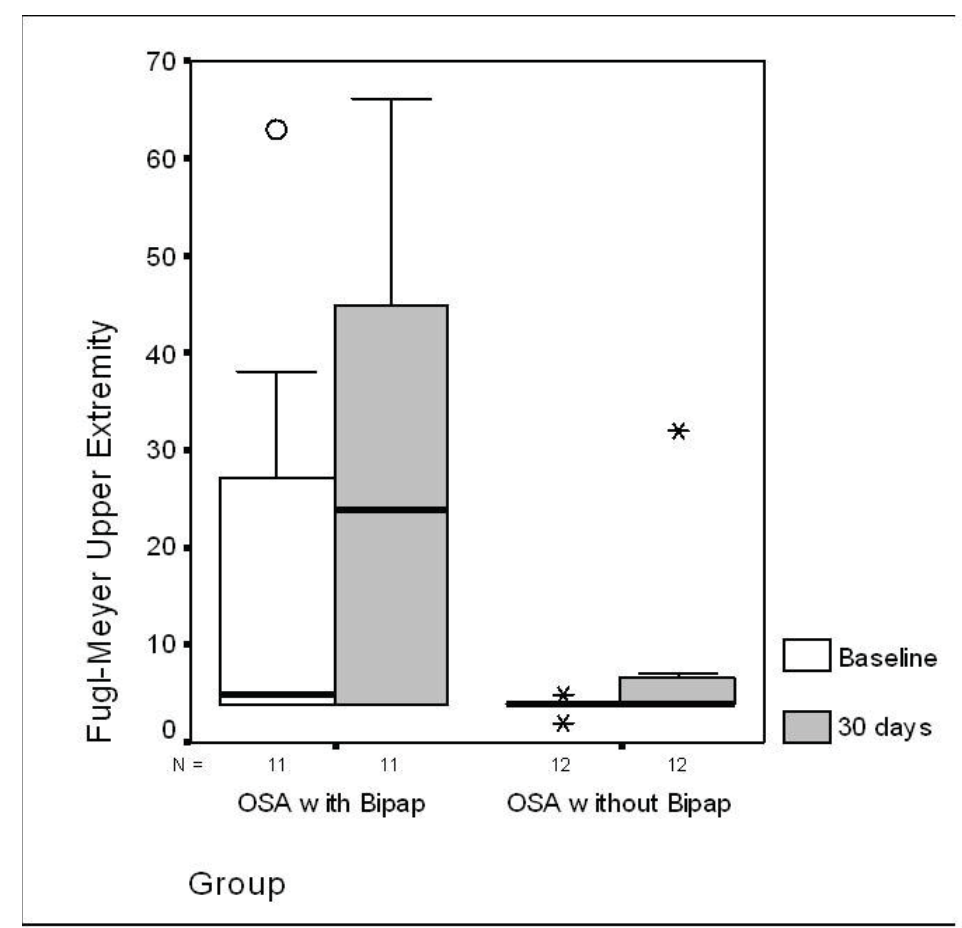

Figure 1. Fugl-Meyer Upper Extremity comparison at baseline and at 30 days between both groups: Obstructive Sleep Apneas treated with BiPAP versus Obstructive Sleep Apneas without BiPAP treatment. Abbreviations: Obstructive Sleep Apnea with BiPAP Treatment (OSA with BiPAP); Obstructive Sleep Apnea without BiPAP Treatment (OSA without BiPAP).

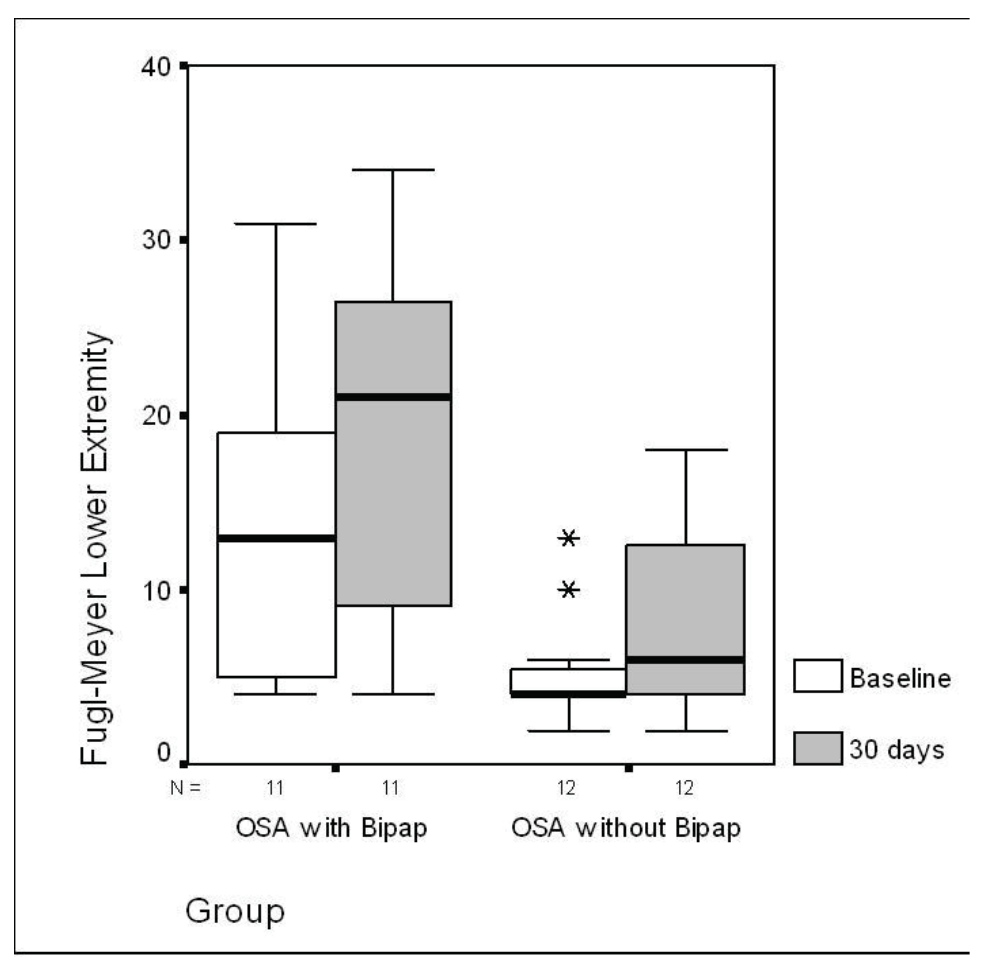

Figure 2. Fugl-Meyer Lower Extremity comparison at baseline and at 30 days between both groups: Obstructive Sleep Apneas treated with BiPAP versus Obstructive Sleep Apneas without BiPAP treatment. Abbreviations: Obstructive Sleep Apnea with BiPAP Treatment (OSA with BiPAP); Obstructive Sleep Apnea without BiPAP Treatment (OSA without BiPAP). 
Table 3. Comparison Between OSA with BiPAP Group Versus OSA Without BiPAP Group

\begin{tabular}{|c|c|c|c|c|}
\hline & OSA w/BiPAP & OSA & Absolute change & p-value \\
\hline \multicolumn{5}{|l|}{ FIM } \\
\hline Baseline day & $43(24.5-50.5)$ & $44(23-49)$ & & \\
\hline Day 30 & $61(57-91.5)$ & $70(38.5-75.7)$ & & \\
\hline \multicolumn{5}{|l|}{ BI } \\
\hline Baseline & $5(0-25)$ & $7.5(0-15)$ & & \\
\hline Day 30 & $50(12.5-57.5)$ & $27.5(5-43.7)$ & & \\
\hline \multicolumn{5}{|l|}{ FMUE } \\
\hline Baseline & $5(4-31)^{*}$ & $4(4-4)^{*}$ & 1 & ${ }^{*} \mathrm{p}=0.027$ \\
\hline Day 30 & $24(4-47)$ & $4(4-6.75)$ & 20 & $* \mathrm{p}=0.05$ \\
\hline \multicolumn{5}{|l|}{ FMLE } \\
\hline Baseline & $13(4-21)^{*}$ & $4(4-5.75) *$ & 9 & ${ }^{*} \mathrm{p}=0.011$ \\
\hline Day 30 & $21(7-27)^{*}$ & $6(4-12.75)^{*}$ & 19 & $* \mathrm{p}=0.019$ \\
\hline \multicolumn{5}{|l|}{ ACE } \\
\hline Baseline & 75 (67-77) & $80(71.5-84.7)$ & & \\
\hline Day 30 & $85(77-92)$ & 80 (79.2-91.5) & & \\
\hline \multicolumn{5}{|l|}{ FAB } \\
\hline Baseline & $10(5.2-14.2)$ & $11(10-13.75)$ & & \\
\hline Day 30 & $12,5(8.7-14.5)$ & $14,5(8.7-17)$ & & \\
\hline \multicolumn{5}{|l|}{ DSF } \\
\hline Baseline & $6(5-7)$ & $5(3.5-6.7)$ & & \\
\hline Day 30 & $6(5-7)$ & $5.5(5-6.7)$ & & \\
\hline \multicolumn{5}{|l|}{ DSB } \\
\hline Baseline & $4(3-4)$ & $3(2.25-4)$ & & \\
\hline Day 30 & $4(3-5)$ & $3(3-4)$ & & \\
\hline
\end{tabular}

Comparison at Baseline and 30 Days in the OSA With BiPAP Group and in the OSA Without BiPAP Treatment (Intragroup Comparison) in the Following Scales: Functional Independence Measure, Barthel Index, Fugl-Meyer Motor Assessment Upper and Lower Extremities, Addenbroke's Cognitive, Examination, Frontal Assessment Battery, Digit Span Subtest of the Wechsler Memory Test. Data are presented as median (percentiles 25-75) unless otherwise stated; * Statistical Significance

OSA with Bipap ( $\mathrm{n}=13)$; OSA without Bipap ( $\mathrm{n}=12)$. Both groups did not differ significantly at baseline regarding gender, supratentorial and infratentorial stroke localization, NIH score, smoking, hypertension and BMI. The number of subjects with coronary heart disease and hypercholesterolemia was higher in the OSA group with BiPAP treatment. These subjects were also younger. Motor, cognitive and neurological functional condition in the group OSA with BiPAP treatment and the group OSA without BiPAP treatment at admission and after 30 days are showed in table 1.No significant differences in AHI index were founding between both groups. After one month of rehabilitation therapy there was a significant improvement in motor and neurological functional scores in both groups. The group treated with BiPAP showed also improvements in the ACE score (see table 2). The group with BiPAP treatment showed significant improvements in the FMUE ( $p=0.05)$; absolute change 19 points (30 days minus baseline) and the FMLE score ( $\mathrm{p}=$ 0.019) absolute change 8 points (30 days minus baseline) compared with the group without BiPAP; absolute change 0 points in FMUE and 2 points in FMLE (Fig. 1, 2). No differences at FAB, Digit span forward and backward were observed in both groups after one month. The comparison between OSA with BiPAP versus OSA without BiPAP treat- 
ment for FIM, BI, Fugl-Meyer upper and lower extremities, ACE, FAB, Digit Span Subtest of the Wechsler Memory Test in treatment and control groups are showed in Table 3.

\section{Discussion}

The use of BiPAP was associated with improvements in motor recovery compared to no-BiPAP use. Furthermore, the evidence is suggestive that BiPAP plays a role in motor recovery after stroke. However, the study design allows for the possibility of a Hawthorne effect associated with the presence or absence of an intervention. So we can only assert that the evidence is suggestive. We found specific improvements in motor scales such as FMUE and FMLE after one month of rehabilitation therapies and BiPAP use. Previous studies suggested that OSA plays a role in impairing recovery from stroke [11], but the impact of BiPAP treatment in the rehabilitation process had not been elucidated. The above observations suggest that BiPAP treatment plays a role in motor recovery of stroke patients.

Sleep-disordered breathing represents both a risk factor and a consequence of stroke and is linked with poorer long-term outcome and increased long-term stroke mortality [5]. Although, sleep-disordered breathing tends to improve spontaneously several weeks post stroke, approximately $50 \%$ of patients still exhibit OSA 3 months after the acute event $[4,6,8]$. The presence of OSA in the setting of stroke is associated with unfavorable clinical course including early neurological worsening, delirium, depressed mood, impaired functional capacity, impaired cognition, and a longer period of hospitalization and rehabilitation.[11, 12, 21, 22]. In view of the high prevalence of sleep apnea in patients with stroke and the effectiveness of BiPAP treatment in improving the quality of life and neurocognitive function, in the reduction of hypersomnia, and the lowering of blood pressure, patients with stroke should be screened for OSA. However, the potential benefit of the BiPAP treatment in the rehabilitation setting has not been completely studied yet. The short term of follow-up and the limited number of subjects may explain the absence of improvements in cognitive test as ACE, Digit spam and FAB between groups.

There are several explanations for the beneficial effect of BiPAP treatment on neurological function following stroke. Apneas and hypopneas cause recurrent cerebral hypoxia that can lead to the production of neuroinhibitory peptides and increased apoptosis of cerebral neurons, both of which could further impair cerebral function [23, 24]. Sleep disruption and excessive daytime sleepiness associated with OSA could also play a role in impairing attention and functional capacity in stroke rehabilitation.

Limitations of the study include the small number of subjects and short-term follow up that preclude possible benefits in cognitive and activities of daily living areas. The clinical team was not informed of the hypothesis but they were not blinded. The trial did not include placebo control. The lack of a sham arm is certainly a limitation of the study. The OSA group without Bipap did not receive any placebo mask simulating BiPAP treatment and could preclude any potential benefits from the placebo effect. There is a possible confounding accounting for the results given the greater age of the OSA patients without Bipap.

\section{Conclusions}

The above observations suggest that PSG evaluation looking for OSA should be part of the standardized evaluation of stroke patients at admission to stroke rehabilitation facilities. The findings suggest that early BiPAP treatment plays a role in the motor recovery of stroke rehabilitation patents. Further research will help elucidate the long-term effects of these strategies.

\section{Acknowledgments}

The authors wish to thank the participants in the trial, all of whom provided valuable contribution: María Elena Mazzola MD, Prof. Horacio Encabo MD from Instituto de Investigaciones Neurologicas Dr. Raul Carrea and Hernan Galimberti MD, FLENI Rehabilitation Institute, Buenos Aires Argentina.

\section{Conflict of Interest}

None declared.

\section{References}

1. Yaggi HK, Concato J, Kernan WN, Lichtman JH, Brass LM, Mohsenin V. Obstructive sleep apnea as a risk factor for stroke and death. N Engl J Med. 2005;353(19):20342041.

2. Quan SF, Gersh BJ. Cardiovascular consequences of sleep-disordered breathing: past, present and future: report of a workshop from the National Center on Sleep Disorders Research and the National Heart, Lung, and Blood Institute. Circulation. 2004;109(8):951-957.

3. Guidelines for management of ischaemic stroke and transient ischaemic attack 2008. Cerebrovasc Dis. 2008;25(5):457-507.

4. Bassetti C, Aldrich MS. Sleep apnea in acute cerebrovascular diseases: final report on 128 patients. Sleep. 1999;22(2):217-223.

5. Bassetti CL. Sleep and stroke. Semin Neurol. 
2005;25(1):19-32.

6. Bassetti C, Aldrich M, Chervin R, Quint D. Sleep apnea in the acute phase of TIA and Stroke. Neurology.1996;47:1167-1173.

7. Wessendorf TE, Teschler H, Wang YM, Konietzko N, Thilmann AF. Sleep-disordered breathing among patients with first-ever stroke. J Neurol. 2000;247(1):41-47.

8. Parra O, Arboix A, Bechich S, Garcia-Eroles L, Montserrat JM, Lopez JA, Ballester E, et al. Time course of sleep-related breathing disorders in first-ever stroke or transient ischemic attack. Am J Respir Crit Care Med. 2000;161(2 Pt 1):375-380.

9. Kushida CA, Littner MR, Morgenthaler T, Alessi CA, Bailey D, Coleman J, Jr., Friedman L, et al. Practice parameters for the indications for polysomnography and related procedures: an update for 2005. Sleep. 2005;28(4):499-521.

10. Practice parameters for the indications for polysomnography and related procedures. Polysomnography Task Force, American Sleep Disorders Association Standards of Practice Committee. Sleep. 1997;20(6):406-422.

11. Yaggi H, Mohsenin V. Obstructive sleep apnoea and stroke. Lancet Neurol. 2004;3(6):333-342.

12. Kaneko Y, Hajek VE, Zivanovic V, Raboud J, Bradley TD. Relationship of sleep apnea to functional capacity and length of hospitalization following stroke. Sleep. 2003;26(3):293-297.

13. Sandberg O, Franklin KA, Bucht G, Eriksson S, Gustafson Y. Nasal continuous positive airway pressure in stroke patients with sleep apnoea: a randomized treatment study. Eur Respir J. 2001;18(4):630-634.

14. Wessendorf TE, Wang YM, Thilmann AF, Sorgenfrei U, Konietzko N, Teschler H. Treatment of obstructive sleep apnoea with nasal continuous positive airway pressure in stroke. Eur Respir J. 2001;18(4):623-629.

15. Mathuranath PS, Nestor PJ, Berrios GE, Rakowicz W,
Hodges JR. A brief cognitive test battery to differentiate Alzheimer's disease and frontotemporal dementia. Neurology. 2000;55(11):1613-1620.

16. Dubois B, Slachevsky A, Litvan I, Pillon B. The FAB: a Frontal Assessment Battery at bedside. Neurology. 2000;55(11):1621-1626.

17. Shuttleworth-Edwards AB. Fine tuning of the digit symbol paired associate recall test for practitioner purposes in clinical and research settings. Clin Neuropsychol. 2002;16(3):232-241.

18. Deutsch A, Braun S, Granger CV. The Functional Independence Measure and the Functional Independence Measure for Children: ten years of development. Crit Rev Phys Med Rehab. 1996; 8:267-281.

19. Mahoney FI, Barthel DW. Functional Evaluation: The Barthel Index. Md State Med J. 1965;14:61-65.

20. Fugl-Meyer AR, Jaasko L, Leyman I, Olsson S, Steglind S. The post-stroke hemiplegic patient. 1. a method for evaluation of physical performance. Scand J Rehabil Med. 1975;7(1):13-31.

21. Hertanu JS, Demopoulos JT, Yang WC, Calhoun WF, Fenigstein HA. Stroke rehabilitation: correlation and prognostic value of computerized tomography and sequential functional assessments. Arch Phys Med Rehabil. 1984;65(9):505-508.

22. Decary A, Rouleau I, Montplaisir J. Cognitive deficits associated with sleep apnea syndrome: a proposed neuropsychological test battery. Sleep. 2000;23(3):369-381.

23. Gozal D, Daniel JM, Dohanich GP. Behavioral and anatomical correlates of chronic episodic hypoxia during sleep in the rat. J Neurosci. 2001;21(7):2442-2450.

24. Douglas RM, Ryu J, Kanaan A, Del Carmen Rivero M, Dugan LL, Haddad GG, Ali SS. Neuronal death during combined intermittent hypoxia/hypercapnia is due to mitochondrial dysfunction. Am J Physiol Cell Physiol. 2010;298(6):C1594-1602. 